\title{
Direct Immunofluorescence of Renal Biopsy: Perspective of an Immunopathologist
}

\author{
${ }^{1}$ Ranjana Walker Minz, ${ }^{2}$ Seema Chhabra, ${ }^{3}$ Kusum Joshi, ${ }^{4} \mathrm{~N}$ Khirwadkar, ${ }^{5}$ Vinay Sakhuja \\ ${ }^{6}$ Neelam Pasricha, ${ }^{7}$ Ranjit Bhardwaj
}

\begin{abstract}
Aims: This study was undertaken to analyze the strength of direct immunofluorescence microscopy in the diagnoses of renal diseases vis-à-vis histopathology. An attempt was also made to present advantages and pitfalls of this age old technique.

Settings and design: A total of 250 consecutive renal biopsies received over a period of 1 year were analyzed. The histopatholgy and direct immunofluorescence slides were reported by two separate pathologists and later compared to reach a final diagnosis.
\end{abstract}

Results: Two cores examined by histopatholgy and direct immunofluorescence microscopy yielded a final diagnosis in $98 \%$ cases. In $2 \%$ of renal biopsies ( 4 biopsies with a 'descriptive' label and 1 case of amyloid like nephropathy), additional diagnostic aids like electron microscopy were required to clinch a conclusive diagnosis. No case of anti-glomerular basement membrane glomerulonephritis or hereditary glomerular disease was observed over 1 year period under review.

Conclusion: Direct immunofluorescence helped to detect IgA nephropathy ( $5 \%$ cases), it incresed the sensitivity of detection of focal segmental glomeulosclerosis (23 more cases) and membranous glomerulopathy (2 more cases). It helped in detection as well as grading of glomerulonephritis in lupus nephritis. Crescentic glomerulonephritis could be further categorized into immune complex and pauci-immune subtypes. Immunofluorescent dye thioflavin $\mathrm{T}$ proved out to be a very sensitive dye for detection of amyloidosis. Membranoproliferative glomerulonephritis/ diffuse proliferative glomerulonephritis cases were less well discriminated by direct immunofluorescence than histopathology. Two percent of renal biopsies ( 2 cases of membranoproliferative glomerulonephritis, 1 case of membranous glomerulopathy, 1 case of mesangioproliferative glomerulonephritis) were false negative on direct immunofluorescence implying technical errors. Thus, correct diagnosis of glomerulonephritis requires direct immunofluorescence

\footnotetext{
${ }^{1,5}$ Professor and Head, ${ }^{2}$ Assistant Professor

${ }^{3}$ Ex-Professor and Head, ${ }^{4}$ Ex-Senior Resident

${ }^{6}$ Ex-Tutor Technician, ${ }^{7}$ Junior Technician

1,2,4,6,7 Department of Immunopathology, Postgraduate Institute of Medical Education and Research, Chandigarh, India
}

${ }^{3}$ Department of Histopathology, Postgraduate Institute of Medical Education and Research, Chandigarh, India

${ }^{5}$ Department of Nephrology, Postgraduate Institute of Medical Education and Research, Chandigarh, India

Corresponding Author: Seema Chhabra, Assistant Professor Department of Immunopathology, Postgraduate Institute of Medical Education and Research, Chandigarh, India, Phone: 01724671791, e-mail: drseemachhabra@gmail.com microscopy in parallel with light microscopic examination and also correlation with clinical features, serological as well as biochemical parameters. In less than $2 \%$ of patients, electron microscopy might be essential.

Keywords: Biopsy, DIF, Kidney.

How to cite this article: Minz RW, Chhabra S, Joshi K, KhirwadkarN, Sakhuja V, Pasricha N, Bhardwaj R. Direct Immunofluorescence of Renal Biopsy: Perspective of an Immunopathologist. J Postgrad Med Edu Res 2015;49(1):10-17.

\section{Source of support: Nil}

\section{Conflict of interest: None}

\section{INTRODUCTION}

The direct immunofluorescence (DIF) is a powerful adjunctive modality for adequate pathologic evaluation of renal biopsies and guides the resolution of the differential diagnosis in a patient with glomerulonephritis (GN). ${ }^{1}$ It is used along with histopathology (HPE) and electron microscopy to correctly diagnose renal diseases. At our 900 bedded tertiary care referral center in North India, we had the unique opportunity to develop DIF facilities separately in the department of immunopatholgy. In the last few years of this practice, great insights have been developed regarding the strength and pitfalls of this technique which are highlighted in this paper. This study is aimed at providing the spectrum of renal conditions observed over a period of 1 year as well as practical difficulties that a practicing nephropathologist would face while using DIF technique as it is practiced in very limited centers in India. This facility needs to be started in the peripheral state/Government and private medical colleges to help detect renal diseases for proper therapy and management. Our paper is an initiative to create an awareness within this vast country regarding this technique.

\section{MATERIALS AND METHODS}

A total of 250 consecutive renal biopsies were analyzed. Two cores of renal biopsies were obtained from each patient by percutaneous needle biopsy. One core for light microscopic examination was fixed in $10 \%$ formalin and embedded in paraffin and then stained routinely with hematoxylin and eosin, periodic acid-schiff (PAS) stain and Jones stain. Second core meant for direct immuno- 
fluorescence microscopy (DIFM) was immediately frozen and embedded in OCT (optimal cutting temperature) compound. The cryostate section were cut and the standard DIF was performed using FITC conjugated antisera against human IgG, IgA, IgM, C3 and fibrinogen. The glomerular and extraglomerular location, intensity and pattern of staining were observed, recorded and reported. Glomerular staining was categorized as mesangial or capillary wall or both. The distribution was described as focal or diffuse and segmental or global. Capillary wall staining was described as granular or linear. The histopatholgy and DIF slides were reported by two separate pathologists and later compared to conclude a final diagnosis. Clinical information was collected from the data submitted by the nephrologists along with biopsy.

\section{RESULTS}

A total of 250 renal biopsies were received in the department of immunopatholgy over a period of 1 year. In them, six biopsies were from postmortem cases and 27 biopsies were referred from outside where corresponding histopathological biopsies and reports were not available with us. In 20 cases, renal biopsies were not adequate for either routine light or DIF microscopic analysis, i.e. in nine cases were nonrepresentative and in remaining 11 cases no glomeruli were seen and only medulla was included in the renal biopsies. So, final analysis was performed in the remaining 197 biopsies. Among 197 cases, 116 were males and 81 were females. Two cores examined by HPE and DIFM yielded a final diagnosis in $98 \%$ cases. In $2 \%$ (5 cases; 4 cases with a 'descriptive' label and 1 case of amyloid like nephropathy) of renal biopsies, a need was felt for additional diagnostic aids like electron microscopy to reach a conclusive diagnosis.

The overall frequencies of different renal diseases are shown in Table 1. It can be seen that primary GN was the most common kidney disease in our patients accounting for 151 (76\%) of all patients. Among the primary GN, focal segmental glomerulosclerosis, FSGS (40\%) was the commonest followed by membranous glomerulopathy (12\%). IgM nephropathy was not found. Most cases of the primary GN were diagnosed in 2nd and 3rd decade of life as shown in Table 2. The most common indication for renal biopsy was nephrotic syndrome (NS) followed by rapidly progressive renal failure. Table 3 shows the clinical syndromes presenting in each histological category.

IgA nephropathy accounted for 5\% (10/197) of total renal biopsy diagnoses and 7\% (10/151) of primary glomerular diseases. The commonest mode of presentation was nephritic syndrome followed by hematuria. Majority of cases fell into class IV ( 5 cases) followed by class II ( 3 cases).

The commonest secondary GN was lupus nephritis ( $9 \%$ ) followed by amyloidosis (2\%) and diabetic nephropathy (1\%). Vascular nephropathy was the least common diagnostic category including one case of malignant nephrosclerosis. Changes of tubulointerstitial nephritis (TIN) were seen in 13 cases. There was no case of hereditary glomerular disease and antiglomerular basement membrane (GBM) GN in this analysis indicating a low incidence of these conditions in our population.

Seventeen cases fulfilled the clinical, serological and immunopathological criteria of lupus nephritis, one was male and others were females. All 17 cases were positive for antinuclear antibodies (ANA). Most common clinical presentation was asymptomatic urinary abnormalities

Table 1: Distribution of all renal diseases observed in 197 native kidney biopsies over a period of 1 year

\begin{tabular}{|c|c|c|c|}
\hline Major category & Renal disease & No. of cases & M:F ratio \\
\hline \multirow[t]{10}{*}{ Primary GN (151) } & Focal segmental glomerulosclerosis & 63 & $42: 21$ \\
\hline & Membranous glomerulopathy & 24 & $17: 7$ \\
\hline & Type I membranoproliferative GN & 11 & $4: 7$ \\
\hline & Minimal change glomerulopathy & 11 & $7: 4$ \\
\hline & IgA nephropathy & 10 & $8: 2$ \\
\hline & Pauci-immune crescentic GN & 10 & $6: 4$ \\
\hline & Immune complex crescentic GN & 8 & $6: 2$ \\
\hline & Diffuse proliferative GN & 8 & $4: 4$ \\
\hline & Chronic GN & 5 & $3: 2$ \\
\hline & Mesangioproliferative GN & 1 & $1: 0$ \\
\hline \multirow[t]{4}{*}{ Secondary GN (25) } & Lupus nephritis & 17 & $1: 16$ \\
\hline & Amyloidosis & 4 & $3: 1$ \\
\hline & Diabetes nephropathy & 2 & $2: 0$ \\
\hline & Hemolytic uremic syndrome & 2 & $1: 1$ \\
\hline \multirow[t]{2}{*}{ Tubulointerstitial nephritis (13) } & Acute tubulointerstitial nephritis & 12 & $6: 6$ \\
\hline & Acute tubular necrosis & 1 & $0: 1$ \\
\hline Vascular nephropathy (1) & Hypertension & 1 & $1: 0$ \\
\hline \multirow[t]{2}{*}{ Miscellaneous (7) } & No significant changes & 6 & $3: 3$ \\
\hline & Amyloid-like nephropathy & 1 & $0: 1$ \\
\hline
\end{tabular}


(11 cases) followed by NS (3 cases). Class IV was the most common pathological subgroup (Table 4).

Direct immunofluorescence findings in kidney biopsies: Of 197 cases, DIF examination was done in all cases, among these $160(81 \%)$ showed positive immunofluorescent findings while 37 (19\%) showed no immune deposits. These 37 cases included 10 cases of minimal change glomerulopathy, five cases of chronic GN, two cases of FSGS, two cases of membranoproliferative GN (MPGN), one case of membranous glomerulopathy, one case of mesangioproliferative GN, 12 cases of TIN, and two miscellaneous cases and one case each of diabetic nephropathy, and acute tubular necrosis (ATN). Extra glomerular immune deposits, i.e. vascular deposits as well as tubular deposits on DIFM were also recorded. C3 reactive vascular deposits were observed in 26 cases including 22 cases of FSGS, two cases of lupus nephritis and two cases of pauci-immune crescentic GN. Granular deposits in renal tubules were found in two cases only; one case of FSGS and one case of IgA nephropathy. Table 5 highlights the frequency of immune deposits in primary GN.

In FSGS, IgM alone was the commonest immunoreac$\operatorname{tant}(95 \%$ cases) found. C3 alone was seen in 30/63 and IgM in combination with $\mathrm{C} 3$ in 25 cases (Fig. 1). Twenty-three biopsies showed normal morphology on HPE but were found to have FSGS on DIFM. Two cases labeled as FSGS on HPE showed no immune deposits on DIF (Table 6). Immunopathologic studies in membranous glomerulopathy showed typical findings (Fig. 2). IgG deposition was found in $23(96 \%)$ cases, followed by IgM $(42 \%)$ and C3 $(42 \%)$. IgA positivity was noted in one case. Only one case

Table 2: Distribution of primary glomerular diseases in different age groups

\begin{tabular}{|c|c|c|c|c|c|c|c|c|c|}
\hline \multirow[b]{2}{*}{ Glomerular diseases } & \multicolumn{9}{|c|}{ Age (years) } \\
\hline & $<10$ & $11-20$ & $21-30$ & $31-40$ & $41-50$ & $51-60$ & $61-70$ & $>80$ & Total \\
\hline Focal segmental glomerulosclerosis & 10 & 24 & 12 & 9 & 6 & 1 & 1 & - & 63 \\
\hline Membranous glomerulopathy & - & 3 & 4 & 6 & 4 & 5 & 2 & - & 24 \\
\hline Type I membranoproliferative GN & 1 & 3 & 3 & 4 & - & - & - & - & 11 \\
\hline Minimal change glomerulopathy & 3 & 5 & 1 & - & - & 2 & - & - & 11 \\
\hline IgA nephropathy & 1 & 6 & 2 & - & 1 & - & - & - & 10 \\
\hline Pauci-immune crescentic GN & - & - & 3 & 1 & 1 & 3 & 1 & 1 & 10 \\
\hline Immune complex crescentic GN & - & 2 & 1 & 3 & 1 & 1 & - & - & 8 \\
\hline Diffuse proliferative GN & 1 & 1 & 2 & 4 & - & - & - & - & 8 \\
\hline Chronic GN & - & - & 2 & 1 & 2 & - & - & - & 5 \\
\hline Mesangioproliferative GN & - & - & - & - & 1 & - & - & - & 1 \\
\hline Total & 16 & 43 & 28 & 28 & 16 & 12 & 4 & 1 & 151 \\
\hline
\end{tabular}

IgA: Immunoglobulin A; GN: Glomerulonephritis

Table 3: Clinical presentation in various renal diseases

\begin{tabular}{|c|c|c|c|c|c|c|c|c|}
\hline Renal disease & Number & $\begin{array}{l}\text { Nephrotic } \\
\text { syndrome }\end{array}$ & $\begin{array}{l}\text { Acute } \\
\text { nephritis }\end{array}$ & $A R F$ & $C R F$ & $A \cup A$ & $R P R F$ & Hematuria \\
\hline Focal segmental glomerulosclerosis & 63 & 51 & - & 3 & 3 & - & 4 & 2 \\
\hline Membranous glomerulopathy & 24 & 21 & - & - & 2 & - & - & 1 \\
\hline Type I membranoproliferative GN & 11 & 9 & - & - & - & 1 & 1 & - \\
\hline Minimal change glomerulopathy & 11 & 11 & - & - & - & - & - & - \\
\hline IgA nephropathy & 10 & 4 & - & - & - & 2 & 1 & 3 \\
\hline Pauci-immune crescentic GN & 10 & - & - & 2 & - & - & 7 & 1 \\
\hline Immune complex crescentic GN & 8 & - & - & - & 1 & - & 7 & - \\
\hline Diffuse proliferative GN & 8 & 1 & 4 & - & - & - & 2 & - \\
\hline Chronic GN & 5 & 1 & - & - & 2 & - & 2 & - \\
\hline Mesangioproliferative GN & 1 & - & - & - & - & - & - & - \\
\hline Lupus nephritis & 17 & 3 & - & - & 2 & 11 & - & 1 \\
\hline Amyloidosis & 4 & 1 & - & 2 & - & - & 1 & - \\
\hline Diabetic nephropathy & 2 & 2 & - & - & - & - & - & - \\
\hline Hemolytic uremic syndrome & 2 & - & - & - & - & - & 2 & - \\
\hline Acute tubular necrosis & 1 & - & - & 1 & - & - & - & - \\
\hline Tubulointerstitial nephritis & 12 & - & 1 & 3 & 3 & - & 5 & - \\
\hline Hypertension & 1 & - & - & - & 1 & - & - & - \\
\hline Amyloid like glomerulopathy & 1 & 1 & - & - & - & - & - & - \\
\hline Descriptive & 6 & 2 & 2 & 1 & 1 & - & - & - \\
\hline
\end{tabular}

ARF: Acute renal failure; AUA: Asymptomatic urinary abnormalities; CRF: Chronic renal failure; IgA: Immunoglobulin A; GN: Glomerulonephritis; RPRF: Rapidly progressive renal failure 


\begin{tabular}{|c|c|c|}
\hline \multirow{3}{*}{$\begin{array}{l}\text { Antinuclear antibody pattern on IIF on } \\
\text { Hep } 2 \text { cell lines }\end{array}$} & Diffuse & 13 \\
\hline & Mixed & 2 \\
\hline & Speckled & 2 \\
\hline \multirow{5}{*}{$\begin{array}{l}\text { Immunoreactant positivity on DIFM of } \\
\text { kidney biopsies }\end{array}$} & $\lg G$ & 16 \\
\hline & $\lg A$ & 12 \\
\hline & $\lg M$ & 16 \\
\hline & C3 & 16 \\
\hline & Fibrinogen & 4 \\
\hline \multirow[t]{5}{*}{ WHO-Class } & I & 1 \\
\hline & II & 3 \\
\hline & III & - \\
\hline & IV & 11 \\
\hline & V & 2 \\
\hline
\end{tabular}

IgA: Immunoglobulin A; IgG: Immunoglobulin G; IgM: Immunoglobulin M

(4.1\%) showed IgM reactive mesangial deposits apart from typical membranous staining pattern. No extraglomerular deposits were reported. Two cases that were labeled as FSGS and normal morphology respectively on HPE were conclusively diagnosed membranous glomerulopathy solely on DIFM (Table 6).

In eight biopsies of minimal change glomerulopathy, immunoglobulis were absent from the glomeruli while 3 biopsies showed low intensity (only $1+$ ) IgM reactive deposits localized to the mesangium. DIFM from cases of IgA nephropathy showed diffuse granular mesangial IgA deposits with the intensity ranging from $2+$ to $4+$ (Fig. 3). In addition, five cases of IgA nephropathy belonging to class $\mathrm{V}$ showed glomerular capillary wall staining as well. $90 \%$ cases showed mesangial deposits of $\operatorname{IgG}$ and $50 \%$ showed that of $\operatorname{IgM}$, although in each case the overall intensity of staining for IgA was as great as that for either of the other immunoglobulin. Mesangial granular deposits of fibrinogen were noted in three cases. Extraglomerular IgA and C3 reactive deposits were seen in one case within renal tubular epithelial cells.

The cases of type I MPGN as well as diffuse proliferative GN (DPGN) that demonstarted positive results on

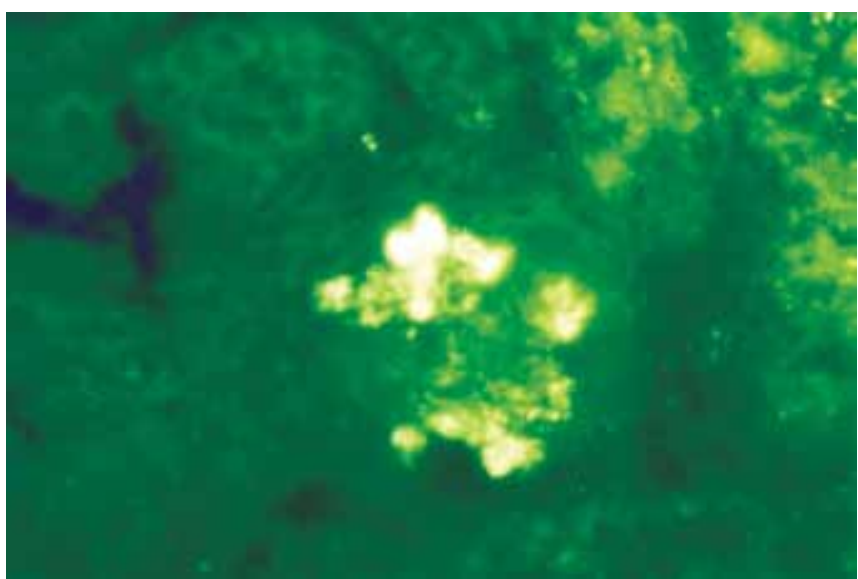

Fig. 1: Direct immunofluorescence photomicrograph of a glomerulus with FSGS showing segmental positivity for IgM (400x)

DIFM, showed IgM and C3 reactive immune deposits in glomerular capillary walls and mesangium in all biopsies (Fig. 4). Only in two cases where DPGN was suggested on findings of diffuse lumpy-bumpy deposits of IgG and/ or $\mathrm{C} 3$ in enlarged lobulated glomeruli, did the diagnosis match histopathological findings. There were five cases that were labeled as MPGN on DIF, were DPGN on histology and 1 case diagnosed as DPGN on DIF showed findings typical of MPGN on histology (Table 6).

Out of a total of 18 cases of crescentic GN, 10 were diagnosed as pauci-immune crescentic GN. Seven of 10 cases showed complete absence of glomerular immunofluorescence staining for immunoglobulins (Fig. 5) while 2 showed $1+$ mesangial staining for IgG, IgM and C3 and were anti-neutrophilic cytoplasmic antibodies (ANCA) negative on serology. None of these showed IgA reactive mesangial deposits. Two of 10 cases were P-ANCA positive while seven cases were ANCA negative. None of these showed positive serum ANA results. Eight cases were given a diagnostic label of immune complex crescentic GN. All these cases showed immune deposits in the glomerular capillary loops as well as in the mesangium with $\mathrm{C} 3$ being the commonest immune-reactant followed by IgM and IgG. Serum ANA and ANCA results were

Table 5: Frequency of immunoreactant positivity in primary glomerular diseases $(n=151)$

\begin{tabular}{llllll}
\hline Renal disease & IgG & IgA & IgM & C3 & Fibrin \\
\hline Focal segmental glomerulosclerosis (63) & 19 & - & 60 & 30 & 20 \\
Membranous glomerulopathy (24) & 23 & 1 & 10 & 10 & - \\
Type I membranoproliferative GN (11) & 8 & 2 & 9 & 9 & 5 \\
Minimal change glomerulopathy (11) & - & - & $3^{*}$ & - & - \\
IgA nephropathy (10) & 9 & 10 & 6 & 7 & 3 \\
Pauci-immune crescentic GN (10) & 1 & - & 2 & 2 & 1 \\
Immune-complex crescentic GN (8) & 7 & 3 & 7 & 8 & 8 \\
Diffuse proliferative GN (8) & 6 & 1 & 8 & - & - \\
Chronic glomerulonephritis (5) & - & - & - & - & - \\
Mesangioproliferative GN (1) & - & - & - & - & - \\
\hline
\end{tabular}

*Only 1+ staining intensity localized to the mesangium. GN: Glomerulonephritis; IgA: Immunoglobulin A; IgG: Immunoglobulin G; IgM: Immunoglobulin M 
negative in all these cases and SLE was also excluded. Five cases of chronic GN did not show immune complexes on immunofluorescent microscopy as the disease becomes quiescent in a fibrotic kidney.

The predominant immune deposits in lupus nephritis (Fig. 6) were IgG, IgM and C3 with equal frequency (16 cases, 94\%) and fibrinogen in crescents in four cases. IgA deposition was seen in 12 of 17 (71\%) renal biopsies including one of the two cases of membranous lupus nephritis (class V). Full house staining for immunoglobulins was observed in 12/17 cases. Vascular deposits with C3 were noted in two cases while tissue ANA with antiserum to IgG (in diffuse pattern similar to that observed in serum ANA testing) was seen in one renal biopsy specimen.

All four cases of amyloidosis showed negative immunofluorescence results with IgG, A, M and C3 but showed $3+$ positivity with thioflavin $\mathrm{T}$ in the glomeruli, tubules and in the walls of the blood vessels (Figs 7A to C). In HUS, on DIFM fibrin could be demonstrated in one of the two cases along with $2+\operatorname{IgG}$ and 2+ IgM mesangial staining. In another case of HUS, no fibrin was demonstrable but segmental IgM and C3 were seen.

One case of diabetic nephropathy showed nonsignificant immunofluorescence findings and was given a 'descriptive' label on DIFM while another case did not show any immune deposits. One case reported as malignant nephrosclerosis on histopathology showed IgM reactive immune deposits in glomerular capillary walls only. None of the 13 cases of TIN showed immune deposits on DIFM.

Out of seven miscellaneous cases, one was diagnosed as amyloid like glomerulopathy, clinically presented as steroid resistant NS and showed mesangial expansion with irregular BM thickening and splitting (confirmed by PAS and Jones stain) on light microscopy. Stain for Congo red and thiofalvin $\mathrm{T}$ was negative. The granular IgG and IgM deposits in membranous pattern were observed on DIFM so lebelled as membranous glomerulopathy. This case highlights the need to use sophisticated tool like electron microscopy. 2/6 miscellaneous cases reported as 'descriptive' on HPE,

Table 6: Immunohistopathological correlation in 197 native renal biopsies

\begin{tabular}{|c|c|c|c|c|c|c|c|c|c|c|c|c|}
\hline & \multirow[b]{2}{*}{ Renal disease } & \multicolumn{10}{|c|}{ Direct immunofluorescence microscopic diagnosis } & \multirow[b]{2}{*}{ Descriptive } \\
\hline & & FSGS & MGN & $\operatorname{IgAN}$ & ICGN & PIGN & MPGN & $D P G N$ & $\begin{array}{l}\text { No } \\
\text { immune } \\
\text { deposits }\end{array}$ & $L N$ & Amyloidosis & \\
\hline \multirow{21}{*}{ 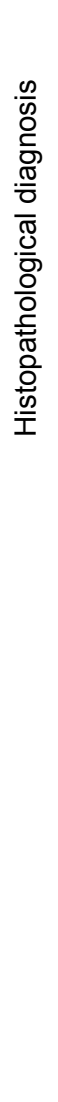 } & FSGS & 36 & - & $3(\mathrm{II})$ & - & - & - & - & 2 & - & - & - \\
\hline & MGN & - & 21 & - & - & - & - & - & 1 & - & - & - \\
\hline & $\begin{array}{l}\text { Mesangipro- } \\
\text { liferative GN }\end{array}$ & - & - & $4(\mathrm{IV})$ & - & - & - & - & 1 & - & - & - \\
\hline & Normal & 23 & 1 & $1(\mathrm{I})$ & - & - & - & - & - & - & - & $2^{*}$ \\
\hline & Crescentic GN & - & - & - & 8 & 9 & - & - & - & - & - & - \\
\hline & $\begin{array}{l}\text { Minimal change } \\
\text { glomerulopathy }\end{array}$ & - & - & - & - & - & - & - & 10 & - & - & - \\
\hline & Type I MPGN & - & - & 1 (IV) & - & - & 8 & 1 & 2 & - & - & - \\
\hline & DPGN & - & - & - & - & - & 5 & 2 & - & - & - & - \\
\hline & Chronic GN & - & - & - & - & - & - & - & 5 & - & - & - \\
\hline & Inadequate & 2 & 1 & - & - & 1 & - & - & - & - & - & - \\
\hline & TIN & - & - & - & - & - & - & - & 12 & - & - & - \\
\hline & ATN & - & - & - & - & - & - & - & 1 & - & - & - \\
\hline & Descriptive & - & - & $1(\mathrm{I})$ & - & - & - & - & - & - & - & $2^{*}$ \\
\hline & $\mathrm{LN}$ & - & - & - & - & - & - & - & - & 17 & - & - \\
\hline & Amyloidosis & - & - & - & - & - & - & - & - & - & 4 & - \\
\hline & $\begin{array}{l}\text { Diabetic } \\
\text { nephropathy }\end{array}$ & - & - & - & - & - & - & - & 1 & - & - & 1 \\
\hline & Hypertension & - & - & - & - & - & - & - & - & - & - & 1 \\
\hline & $\begin{array}{l}\text { Hemolytic } \\
\text { uremic } \\
\text { syndrome }\end{array}$ & - & - & - & - & - & - & - & - & - & - & 2 \\
\hline & $\begin{array}{l}\text { Amyloid like } \\
\text { nephropathy }\end{array}$ & - & 1 & - & - & - & - & - & - & - & - & - \\
\hline & Ischemia & - & - & - & - & - & - & - & 1 & - & - & - \\
\hline & Mesangiolysis & - & - & - & - & - & - & - & 1 & - & - & - \\
\hline
\end{tabular}

*These four cases remained undiagnosed. A need for EM was felt. ATN: Acute tubular necrosis; DPGN: Diffuse proliferative GN; FSGS: Focal segmental glomerulosclerosis; GN: Membranous glomerulopathy; ICGN: Immune complex GN; Ig AN: IgA nephropathy; LN: Lupus nephritis; MGN: Glomerulonephritis; MPGN: Membranoproliferative GN; PIGN: Pauci-immune GN; TIN: Tubulointerstitial nephritis 
one showed ischemic changes and another showed mesangiolysis, and were completely negative on DIFM. The remaining 4 cases had a varied symptomatology and

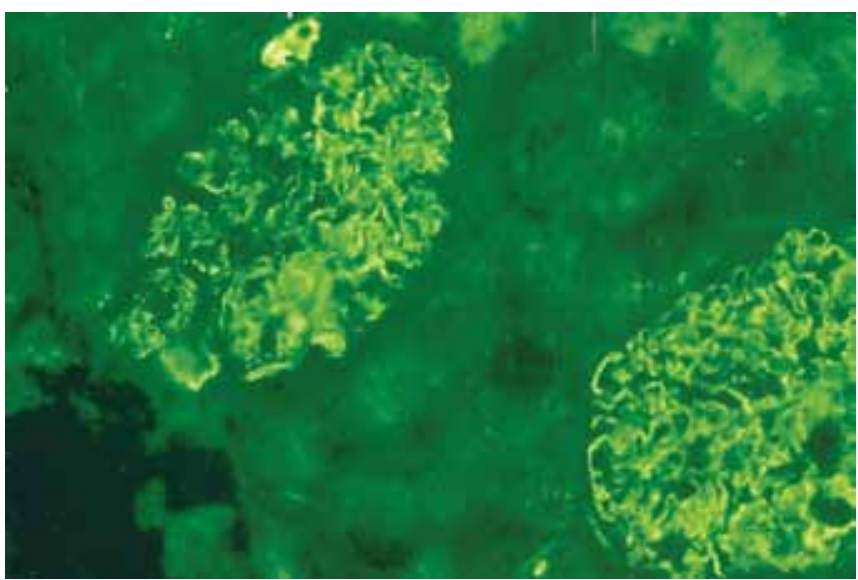

Fig. 2: Direct immunofluorescence photomicrograph from membranous glomerulopathy showing intense $(3+)$ granular deposits of immunoglobulin $\mathrm{G}$ along the entire glomeruler basement membrane. Mesangial areas are free of deposits $(400 x)$

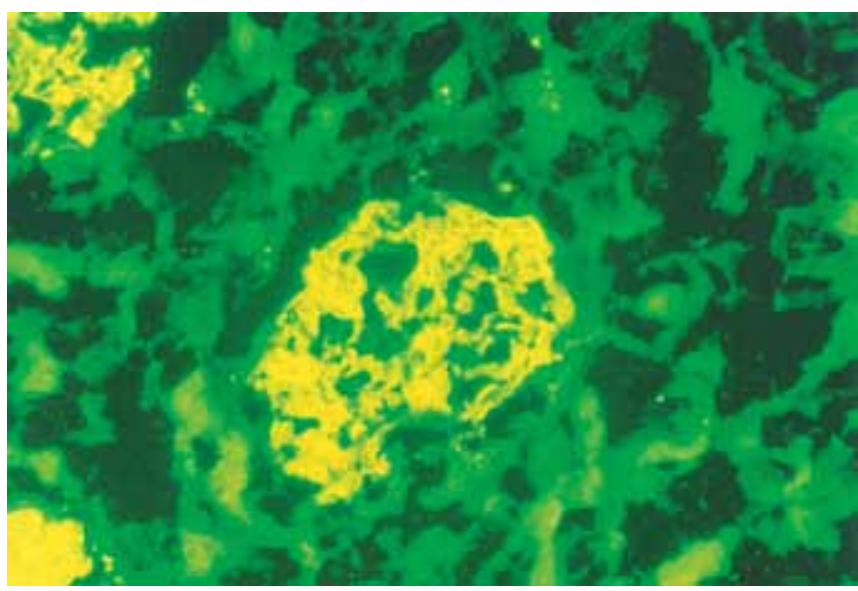

Fig. 3: Direct immunofluorescence photomicrograph showing typical mesangial deposits of immunoglobulin A in renal biopsy of immunoglobulin A nephropathy (400x)

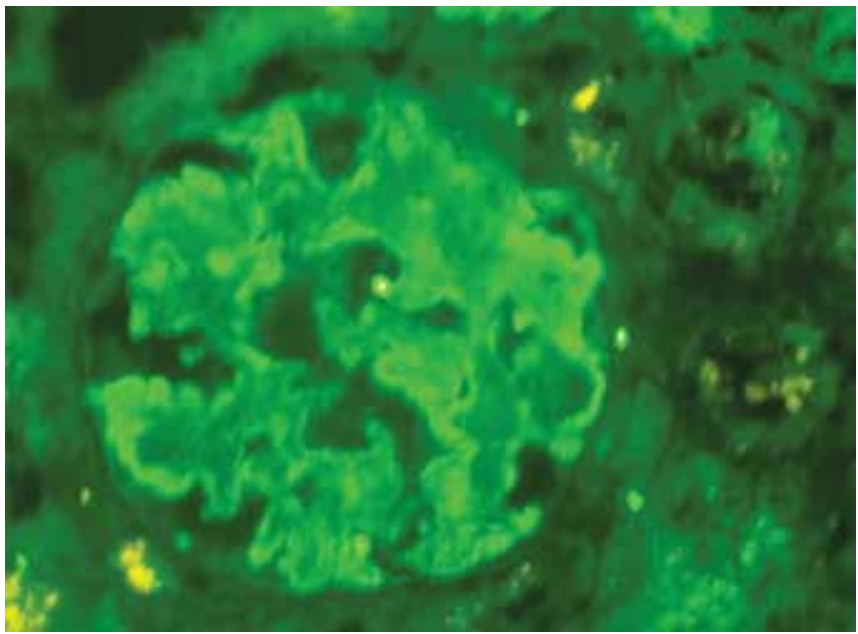

Fig. 4: Direct immunofluorescence photomicrograph from MPGN type I showing moderately intense glomerular mesangial and capillary wall staining for $\mathrm{C}_{3}$ with lobular accentuation (400x) did not show any significant changes with two biopsies being entirely normal on HPE and demonstrated nonspecific immune deposits on DIFM. These biopsies were finally given a descriptive label. No conclusive diagnosis could be made in these four cases.

\section{DISCUSSION}

World over renal biopsy histopathology is known to facilitate diagnosis and registry of disease pathology. The adjunctive technology of DIFM on another biopsy core was first reported from India from department of Immunopathology, PGIMER in 1980 by Sehgal et al. ${ }^{2}$ She documented the presence of immune complexes in the postmortem kidneys of patients dying of viral hepatitis. In 1995, the same group described IgA nephropathy in North India. ${ }^{3}$ Date et $\mathrm{al}^{4}$ in 1987 from CMC Vellore described the largest series of renal biopsies of which 285 had immunofluorescence findings. Presently experience of renal biopsy diagnosis has been described from centers in Kerala, Hyderabad, Chandigarh, New Delhi and West Bengal. ${ }^{4-10}$ The need of the hour is to have renal biopsy diagnostic units in government/private medical colleges to facilitate timely diagnosis and treatment of renal diseases.

NS was the most frequent clinical presentation at all age groups accounting for $54.3 \%$ of all cases. ${ }^{4-15}$ This is in accordance with the many other studies published around the world including Asiatic countries. ${ }^{4-15}$ Overall a male predominance is noted except lupus nephritis where an increased prevalence was found in females, again conforming to the recently published studies worldwide showing a similar pattern. ${ }^{4-15}$ The most common renal disease in our series was FSGS (40\%) followed by membranous glomerulopathy, MPGN/DPGN, MCD. This observation is slightly deviating from other previous

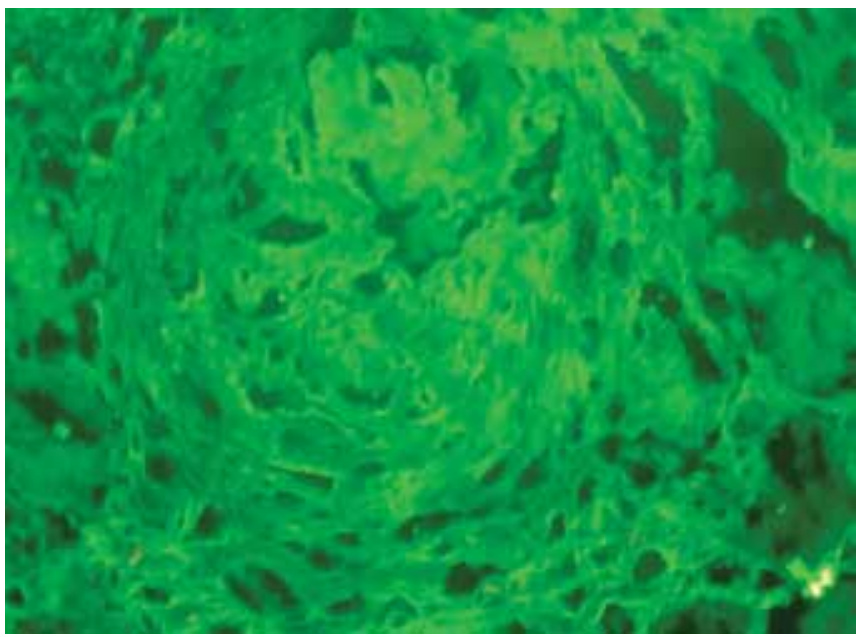

Fig. 5: Direct immunofluorescence photomicrograph of a glomerulus with pauci-immune crescentic glomerulone phritis showing complete absence of immunofluorescence staining for immunoglobulins (400x) 
studies from our country where MCD was found to be the commonest followed by FSGS. ${ }^{40}$ In comparison to European countries as well as some Asian countries, IgA nephropathy was found to be relatively uncommon in this region of the world with a prevalence ranging from 5 to $14 \%{ }^{16-22}$ Lupus nephritis has been documented as the most common secondary GN almost across the world. ${ }^{4-15}$

DIFM has distinct advantages. Diseases like IgA nephropathy, IgM nephropathy, C1q nephropathy and anti-GBM glomerulonephritis can only be diagnosed by immunohistology, whereas the diagnosis of other diseases is confirmed and refined by DIFM. It is of a great help in categorizing crescentic GN into immune complex GN and pauci-immune GN thus facilitating recognition of ANCA associated nephropathy. ${ }^{23}$ It picks up early and late cases of idiopathic membranous glomerulopathy. Diagnostic features in idiopathoic membranous glomerulopathy are very well appreciated on light microscopy except in early disease when capillary walls appear normal and in late disease when GBM thickening is relatively nonspecific by light microscopy. This subtlety of light microscopic changes in some cases of membranous glomerulopathy poses a difficulty in distinguishing it from some other causes of NS including minimal change glomerulopathy

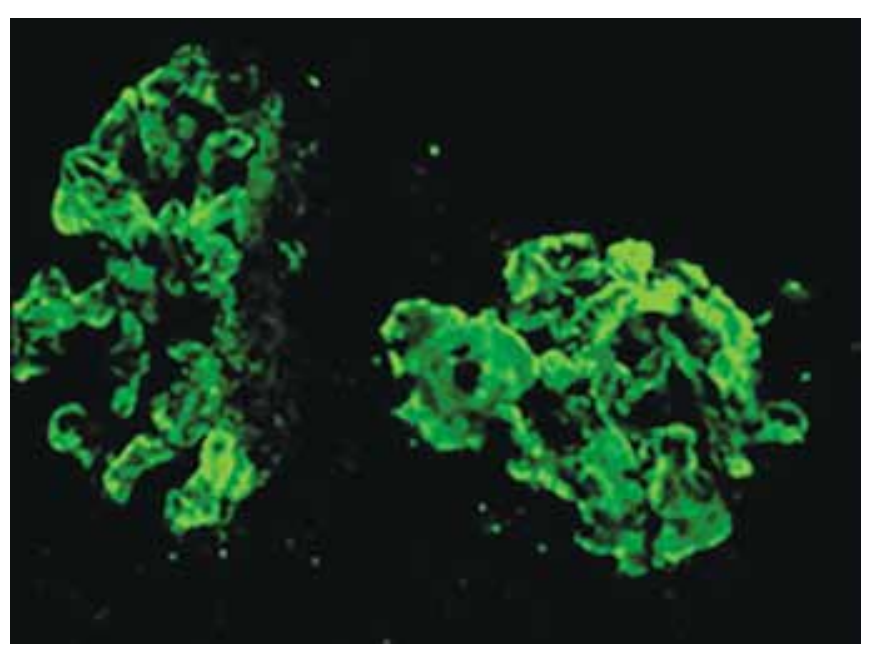

Fig. 6: Direct immunofluorescence photomicrograph from lupus nephritis-IV showing strong staining for immunoglobulin $M$ in the glomerular mesangium and peripheral capillary loops (400x) and certain forms of chronic GN. In these early and late cases, uncertainty regarding the diagnosis of membranous glomerulopathy can be resolved using DIFM. ${ }^{24}$ It also picks up a larger number of early FSGS cases. Recognizing a segmental glomerular lesion is critical as no matter how small or focally distributed FSGS is, removes the patient from the category of minimal change glomerulopathy and alters the therapeutic implication. The presence of 'significant' glomerular immune deposits on DIFM excludes a diagnosis of minimal change glomerulopathy. It aids in grading of lupus nephritis. It is of great benefit in diagnosing amyloidosis as thioflavin $\mathrm{T}$ immunofluorescence is much more sensitive than congophilia in detecting small deposits of amyloid. ${ }^{25}$

This technique has numerous pitfalls and difficulties too, which should be borne in mind while using it for renal disease diagnostics. DPGN/type I MPGN is a difficult issue to be resolved on DIFM without the knowledge of histopathology, as the two lesions have near identical immunofluorescence findings, i.e. coarsely granular capillary wall staining with C3 and/or Igs. However, it is known that MPGN being a heterogeneous disease remains a difficult diagnostic dilemma for the pathologist with sometimes electron microscopy serving as a valuable adjunct. It cannot distinguish between periglomerular fibrosis and crescents. It can be processed only in the sophisticated laboratories that are proficient in the performance and interpretation of these tests. It requires thoroughly trained team comprising of pathologist and a technologist in a specialized lab having facilities of cryostat for cutting frozen sections, $-20^{\circ} / 80^{\circ} \mathrm{C}$ deep freezers to store these sections until staining and a fluorescence microscope to report the DIF findings. The renal biopsy is best examined in an unfixed state. It is fragile and should be transported in ice within 1 hour of biopsy as it is prone to drying. Also, it gets fixed on exposure to formalin vapors so extreme care is required in handling the biopsy. The fluorescence staining quenches rapidly on exposure to light, more so under the UV light of the fluorescence microscope, demanding a fast reporting
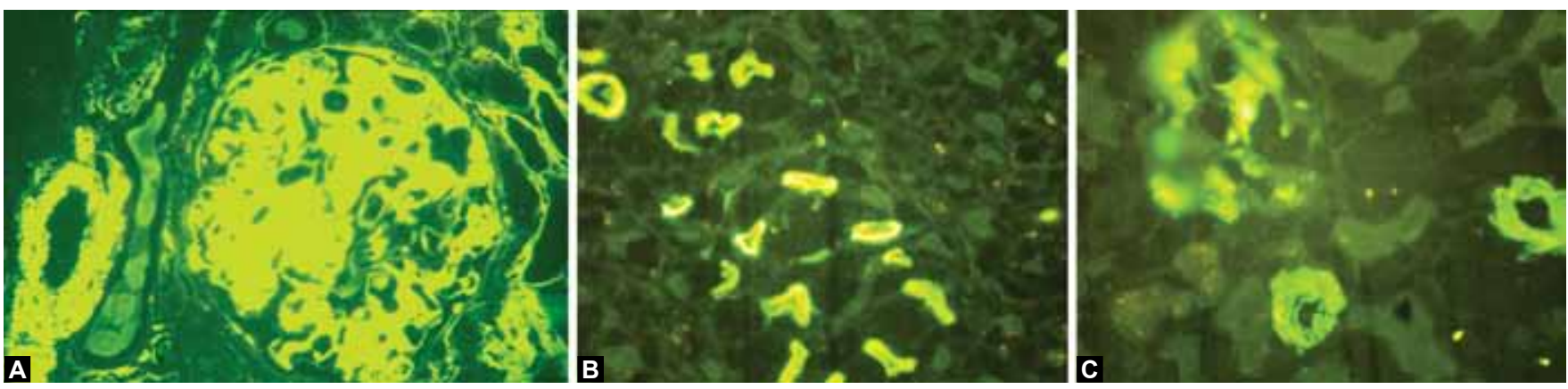

Figs 7A to C: Direct immunofluorescence photomicrograph from amyloidosis showing 4+ thioflavin T positivity: (A) in the glomerulus which is almost obliterated by amyloid deposits along with afferent blood vessel with amyloid deposits in its wall, (B) in the tubules and (C) in the interstitial blood vessels (400x) 
and documentation of findings using a digital camera. No long-term storage period is ideal for reporting of DIF stained renal biopsy slides. Dried and/or fixed biopsies are reported unfit for DIFM. In our study also there were 6 cases of primary GN where no immune complex deposits were seen on DIF. Here in the inability to demonstrate immune complexes in classical cases of histopathology proven MPGN (2 cases), FSGS (2 cases) membranous GN (1 case), and mesangioproloferative GN (1 case) was attributed to technical problems, namely exposure to formalin vapors/drying up of the biopsies sent for DIF examination. This again highlights the importance of experience in handling of unfixed kidney biopsies, meant for DIF studies.

Four cases, where descriptive nonspecific findings were present on both HPE and DIFM in spite of significant clinical symptoms, remained undiagnosed. EM in these cases might have helped by excluding early membranous disease, early amyloid, abnormal basement membrane in normal looking glomeruli by HPE. ${ }^{26}$ Sometimes, the follow-up and clinical behavior of the patient provides the true diagnosis. The present study is a retrospective one, where in no follow-up data were available and this also is a gross limitation in our analysis.

Thus, the correct diagnosis of glomerulonephritis requires DIFM in parallel with light microscopic examination and also correlation with clinical features as well as serological and biochemical parameters.

\section{REFERENCES}

1. Das RK, Saleh AF, Kabir AN, Talukder SI, Kamal M. Immunofluorescence studies of renal biopsies. Dinajpur Med Col J 2008;1(1):8-13.

2. Sehgal S, Dutta BN, Aikat BK, Chugh KS, Dutta DV. Renal immune complexes in viral hepatitis. An autopsy study. Clin Exp Immunol 1980;40(3):453-455.

3. Sehgal S, Datta BN, Sakhuja V, Chugh KS. Primary IgA nephropathy: a preliminary report. Indian J Pathol Microbiol 1995; 38(3):233-237.

4. Date A, Raghavan R, John TJ, Richard J, Kirubakaran MG, Shastry JC. Renal disease in adult Indians: a clinicopathological study of 2,827 patients. QJ Med 1987;64(245):729-737.

5. Chugh KS, Sakhuja V. Glomerular disease in the tropic. Am J Nephrol 1990;10(6):437-450.

6. Balakarishnan N, John GT, Korula A. Spectrum of biopsy proven renal disease and changing trends at a tropical tertiary care centre 1990-2001. Indian J Nephrol 2003;13:29-35.

7. Narasimhan B, Chacko B, John GT, Korula A, Kirubakaran MG, Jacob CK. Characterization of kidney lesions in Indian adults: towards a renal biopsy registry. Nephrol 2006;19(2): 205-210.

8. Das U, Dakshinamurty KV, Prayaga A. Pattern of biopsy proven renal disease in a single centre of South India: 19 years experience. Indian J Nephrol 2011;21(4):250-257.
9. Siddappa S, Kowsalya R, Mythri KM. IgA nephropathy in a tertiary care centre from South India. Indian J Nephrol 2011; 21(4):230-234.

10. Golay V, Trivedi M, Abraham A, Roychowdhary A, Pandey R. The spectrum of glomerular diseases in a single center: a clinicopathological correlation. Indian J Nephrol 2013;23(3): 168-175.

11. Mubarak M, Kaji JI, Naqvi R, Ahmed E, Akhter F, Naqvi $\mathrm{SA}$, et al. Pattern of renal diseases observed in native renal biopsies in adults in a single centre in Pakistan. Nephrology 2011;16(1):87-92.

12. In Joo Choi, Hyeon Joo Jeong, Dae Suk Han. An analysis of 4,514 cases of renal biopsy in Korea. Yonsei Med J 2001;42(2): 247-254.

13. Chang JH, Kim DK, Kim W. Changing prevalence of glomerular disease in Korean adults: a review of 20 years of experience. Nephrol Dial Transplant 2009;24(8):2406-2410.

14. Research group on progressive chronic renal disease. Nationwide and long term survey of primary glomerulonephritis in Japan as observed in 1,850 biopsied cases. Nephron 1999;82(3):205-213.

15. Gesualdo L, Maria A, Francesco L. The Italian experience of the national registry of renal biopsies. Kidney Int 2004;66(3): 890-894.

16. Bhuyan UN, Dash SC, Srivastava RN, Tiwari SC, Malhotra KK. IgA associated glomerulonephritis. J Assoc Physicians India 1992;40(5):310-313.

17. Bakhsi A, Minz RW, Joshi K, Sakhuja V. Clinicopathological spectrum of IgA nephropathy: a 5-year study. Indian J Nephrol 2003;13:131-134.

18. Vaniker AV, Kanodia KV, Patel RD, Trivedi HL. Primary immunoglobulin (Ig) A nephropathy in Western India. Indian J Nephrol 2005;15:227-231.

19. Chacko B, John GT, Neelakantan N, Korula A, Balakrishnan N, Kirubakaran MG, Jacob CK. Presentation, prognosis and outcome of IgA nephropathy in Indian adults. Nephrology (Carlton) 2005;10(5):496-503.

20. Kumari CB. IgA nephropathy in Kerala, India: a retrospective study. Indian J Pathol Microbiol 2009;52(1):14-16.

21. Minz RW, Bakshi A, Chhabra S, Joshi K, Sakhuja V. Role of myofibroblasts and collagen type IV in patients of IgA nephropathy as markers of renal dysfunction. Indian J Nephrol 2010;20(1):34-39.

22. Mittal N, Joshi K, Rane S, Nada R, Sakhuja V. Primary IgA nephropathy in north India: is it different? Postgrad Med J 2012;88(1035):15-20.

23. Gupta R, Singh L, Sharma A, Bagga A, Agarwal SK, Dinda AK. Crescentic glomerulonephritis: a clinical and histomorphological analysis of 46 cases. Indian J Pathol Microbiol 2011;54(3):497-500.

24. Heptinstall RH. Idiopathic membranous, membranoproliferative and lobular glomerulonephritis. In: Heptinstall RH, editor. The pathology of the kidney. Boston: Little, Brown and Company; 1964. p. 393-417.

25. Herrera GA. Renal manifestations in plasma cell dyscrasia: an appraisal from the patient's bedside to research laboratory. Ann Diagn Pathol 2000;4(3):174-180.

26. Pearson JM, McWilliam LJ, Coyne JD, Curry A. Value of electron microscopy in diagnosis of renal disease. J Clin Pathol 1994;47(2):126-128. 